\title{
EDUCATION AND TRAINING Are conflict of interest declarations appropriate to allow sufficient consideration of potential bias in presentations?
}

\author{
Authors: William Crawford, ${ }^{\mathrm{A}^{*}} \mathrm{C}$ Fielder Camm, ${ }^{\mathrm{B}^{*}}$ Ishika Prachee, ${ }^{\mathrm{A}}$ Jack Olivarius-McAllister, ${ }^{\mathrm{A}}$ \\ Matthew R Ginks ${ }^{\mathrm{C}}$ and Edward D Nicol ${ }^{\mathrm{D}}$
}

\section{Background}

Potential conflicts of interest (CoI) are common in medical research, necessitating the use of $\mathrm{CoI}$ declarations. There is currently no consensus document or external authority guiding CoI declarations in conference settings, resulting in declarations of variable quality and utility.

\section{Methods}

We explored four CoI declaration parameters (sufficient slide display time; the presence of any verbal explanation pertaining to relevant CoI; the use of an adequate font size; and whether the nature and relevance of the CoI was described). Parameters were graded from one to three points, with the sum of parameters providing an overall declaration quality out of 12. We then applied this scoring system to recordings of presentations from the British Cardiovascular Society (BCS) annual conference 2018 which were available online.

Results

Sixty-nine presentations were suitable for inclusion, of which $47(68 \%)$ contained a CoI statement. Thirty-six of the $47(77 \%)$ presentations declared that they had no CoI. In the remaining $11(23 \%)$ with reported CoI, the median time spent displaying CoI was 1 second (interquartile range (IQR) 0.7-3.3). The median quality score for presentations was 7 (IQR 6-10).

Conclusion

This study demonstrates utility in considering aspects of CoI declarations at conferences to improve transparency.

KEYWORDS: Conflicts of interest, disclosure, medical education

DOI: $10.7861 /$ fhj.2020-0018

Authors: ${ }^{\text {A }}$ medical student, University of Oxford, Oxford, UK; ${ }^{B}$ cardiology registrar, Royal Berkshire Hospital, Reading, UK; ${ }^{C}$ consultant cardiologist, Oxford University Hospitals NHS Foundation Trust, Oxford, UK; ${ }^{\mathrm{D}}$ consultant cardiologist, Royal Brompton Hospital, London, UK and honorary senior lecturer, National Heart and Lung Institute, London, UK; " authors are joint first co-authors

\section{Introduction}

Industry involvement is common within medical research, producing potential conflicts of interest (CoI). ${ }^{1}$ Industry interaction and CoI are associated with inappropriate prescribing, disproportionate publication of outcomes that favour sponsors, and poor research methodology. ${ }^{2-4}$ Potential CoI are particularly common in cardiology. ${ }^{5}$

Recent initiatives have been introduced in an attempt to increase transparency between clinicians and industry partners, such as the Physician Payments Sunshine Act in the USA, the Disclosure UK database and the adoption of the standardised International Committee of Medical Journal Editors form, used by many journals. ${ }^{6-8}$ Conference presentations are an important medium for communicating medical research. Despite advances made in the publishing world, there is currently no external authority or consensus document to guide how $\mathrm{CoI}$ declarations are presented at medical conferences.

Previous studies have demonstrated that a large proportion of conference presentations do not contain CoI declarations, or that declarations are displayed for too short a time period to be read or understood. ${ }^{9,10}$ Although many conferences have made CoI declaration slides compulsory, declarations are often brief and therefore of debateable value. ${ }^{10}$ As far as we are aware, there has been no specific analysis of the quality of CoI declarations, such as time spent making declarations with respect to the number of words presented on the CoI slide. Analysis of further quality factors, such as font readability and the presence of verbal explanation are also lacking from other studies.

This pilot study aimed to investigate the overall quality of $\mathrm{CoI}$ declarations in a UK cardiology conference setting; specifically, to determine whether cardiologists at a national conference in the UK are presenting their potential CoI so that they can be appropriately understood by conference attendees.

\section{Methods}

\section{Data}

Recordings of presentations from the British Cardiovascular Society (BCS) annual conference 2018 were available through the BCS website. Presentations were excluded if they were opening/ closing sessions, award presentations, motivational talks from 
Table 1. Scoring metric used to quantify quality of conflict of interest declarations in a conference setting

\begin{tabular}{|c|c|c|c|c|}
\hline Points awarded & Font size & Time displayed & Verbal explanation & Level of detail \\
\hline 0 & No text present & No declaration displayed & No comment & No declaration \\
\hline 1 & $<24 \mathrm{pt}$ & $<300 \mathrm{~ms} /$ word & 'These are my conflicts' or similar & Conflicting organisations named \\
\hline 2 & $24-28 \mathrm{pt}$ & $300-500 \mathrm{~ms} /$ word & $\begin{array}{l}\text { Verbal explanation of some } \\
\text { conflicts mentioned }\end{array}$ & $\begin{array}{l}\text { Conflicting organisations named } \\
\text { plus nature of conflict explained }\end{array}$ \\
\hline 3 & $>28 p t$ & $>500 \mathrm{~ms} /$ word & $\begin{array}{l}\text { Verbal explanation of all conflicts } \\
\text { mentioned }\end{array}$ & $\begin{array}{l}\text { Conflicting organisations named, } \\
\text { nature of conflict explained plus } \\
\text { relevance of conflict explained }\end{array}$ \\
\hline
\end{tabular}

non-medical speakers or if slides were unavailable. Presentations were reviewed; time taken to declare potential CoI, the number of words present on the declaration slide and the presence of any accompanying verbal explanation were all noted. The font size used to declare potential conflicts was determined by calculating the height of capitalised letter as a percentage of the overall slide height, controlling for aspect ratios. Font sizes were expressed as point size relative to the default BCS declaration font, which was calculated as a percentage of the overall slide height, and arbitrarily assigned a value of $28 \mathrm{pt}$. Presenters without a CoI declaration were cross-referenced with The Association of the British Pharmaceutical Industry (ABPI) Disclosure UK database to determine whether any payments had been disclosed through this database.

\section{Quality score}

A 12-point metric was developed to quantify the quality of declaration statements. Points were awarded in four categories; including the presence of a verbal explanation, the level of detail provided, whether the declaration slide was displayed for an appropriate amount of time and whether the font size used was appropriate (Table 1). Zero to three points were allocated for each of these declaration characteristics.

\section{Statistical analysis}

Results were analysed using SAS 9.3 (SAS Institute, Cary, USA). Frequencies are presented as $n(\%)$ and continuous variables as median (interquartile range (IQR)).

\section{Results}

\section{Declarations at BCS}

Eighty-four presentations were available for review. Of these, 68 met the inclusion criteria. Of these $68,47(69 \%)$ presentations contained a CoI statement; 22 presentations ( $47 \%$ ) provided declarations on the title slide, while 26 (55\%) provided declarations on a dedicated slide (one presentation provided declarations on both slides). Twenty-one (31\%) of presentations meeting the inclusion criteria did not provide a statement outlining whether they had any CoI present (Fig 1). Of the 21 individuals without a CoI statement on their presentation, 11 (52\%) had some industry payment recorded on the ABPI Disclosure UK database occurring in 2018 or earlier.

Of those with a CoI statement, 36 presentations declared that they had no CoI (77\%). Of those with reported CoI ( $n=11 ; 23 \%)$, the median number of conflicts was 4 (IQR 3-13).

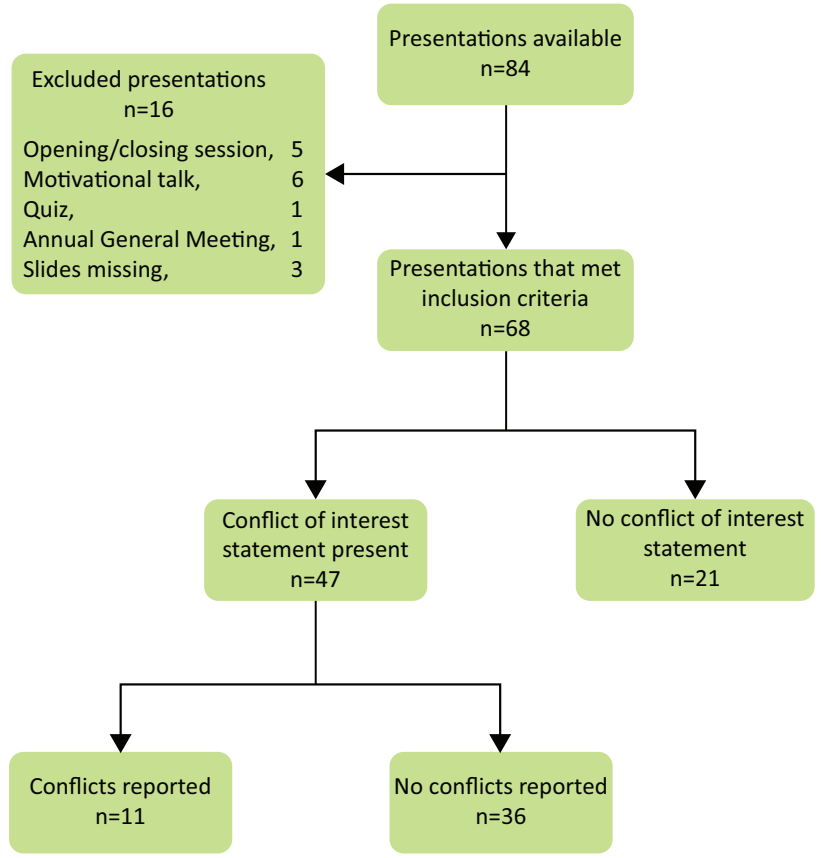

Fig 1. Flowchart detailing selection of included presentations for this analysis.

\section{Overall disclosure quality}

Twenty-one presentations had no CoI disclosure slide and thus had a quality metric score of 0 . Of those with CoI statements, the median score was 9 (IQR 7-11). In presentations without a declared CoI, the median score was 9.5 (IQR 8-11.25). In presentations with a declared CoI, the median score was 7 (IQR 6-9.5). Two presentations with CoI (18\%) scored a maximum possible 12 points, while one presentation scored 5 points.

\section{Time spent on declarations}

In those without declared conflicts, presenters spent a median of 6 seconds on the declaration slide ( $n=35 ;$ IQR $2-14$ ). In those with conflicts, the median duration was 10 seconds ( $n=12$; IQR 4-20) and the median time per conflict was 1 second (IQR 0.7-3.3). There was a median of 19 words on declaration sides in presenters with reported CoI (IQR 8-37). The median reading speed required to completely read the declaration statements of presenters with stated conflicts was 0.3 seconds per word (IQR 0.2-1.1). Three of 
the 11 presenters $(27 \%)$ with reported conflicts displayed their declaration statement for a time such that the average reader could not completely read it. ${ }^{11}$ The median reading speed required to completely read the declaration statements of presenters without stated conflicts was 0.9 seconds per word (IQR 0.5-2.9). Two of the 36 presenters ( $6 \%$ ) without reported conflicts displayed their declaration statement for a time such that the average reader could not completely read it.

\section{Verbal declarations}

Of those declaring no CoI, only 21/36 (58\%) made any reference to the declaration. One presenter stated they had no CoI on the declaration slide but verbally reported that they had received travel reimbursement. Of those with reported CoI, five (45\%) provided no verbal explanation or only stated that 'these are my conflicts/disclosures'.

\section{Font size}

The mean font size used in CoI declarations was $94.8 \%$ (standard deviation (SD) 25.4) of the default BCS font. This equates to an average font size of approximately $26 \mathrm{pt}$, compared with the conference's default size of $28 \mathrm{pt}$. When a CoI was not present, the average font size used was $98.8 \%$ (SD 27.3) of the default, equating to size 27.5 pt font. When a CoI was present, the average font size used was $84.9 \%$ (SD 17.4) of the default, equating to size 23 pt font. Six presentations with relevant $\mathrm{CoI}$ used a font size less than $24 \mathrm{pt}$.

\section{Discussion}

CoI declarations are an essential tool to improve research transparency, allowing audiences to determine whether specific factors may increase the likelihood of bias. At the conference analysed in this pilot study, a large proportion (31\%) of presentations did not include a CoI declaration. Of those with CoI declarations presented, a quarter reported potential conflicts. A minority of this subset appropriately declared their $\mathrm{CoI}$, with only two presentations receiving full marks on the quality metric.

\section{The merit of compulsory conflict declarations}

This study demonstrates that CoI declarations are not consistently present in conference presentations. Interestingly, we observed half of presenters without a CoI declaration had recorded payments on the ABPI Disclosure UK database. This suggests there are a proportion of CoIs which go unreported.

The lack of consistency regarding CoI disclosures has been echoed by other studies. Grey et al analysed presentations from five conferences representing various specialties (one of which was a cardiovascular-themed conference), and demonstrated that only $71 \%$ of presentations contained a CoI declaration. ${ }^{9} \mathrm{CoI}$ statements were present in $95 \%$ of the talks given at the European Society of Cardiology Annual Congress 2016, a large international conference. This conference mandates that $\mathrm{CoI}$ declarations are compulsory for all presenters. ${ }^{10}$ This finding suggests that mandating inclusion of a CoI slide may improve the proportion of presenters providing a description of their potential conflicts.

\section{Improving the quality of conflict declarations}

Presenters with conflicts spent a median of 10 seconds on their declaration slides. While this may appear substantial, the median time per conflict was only 1 second. Furthermore, approximately one-quarter of presenters with a conflict did not display their declaration for long enough for the average person to read the statement. This severely reduces the utility of the CoI declaration. Presentations included in our study spent more time declaring CoI than in other conferences. Previous data have demonstrated that presenters with any conflict spent median time of 2-3 seconds their CoI slide. ${ }^{9,10}$ The assessment of time-per-word is an important addition to the literature on CoI statements. Time spent on the declaration slide is a proxy to establish whether the viewer has enough time to evaluate potential conflicts. However, unless this is indexed to text volume, this value will not truly indicate whether presentation duration is appropriate, or the font readable. This finding highlights a potential issue with conferences mandating a predefined minimum period for display of CoI disclosures, as this does not take text size into account.

In addition to declaration slide duration, many presentations had additional aspects that made it more difficult for audience members to appreciate potential conflicts. Verbal acknowledgements of potential conflicts were present in fewer than half of presentations with $\mathrm{CoI}(n=5 ; 45 \%)$. Furthermore, a font size substantially smaller than the default was used in over half of those with CoI. To our knowledge, no study has analysed font size used to declare CoI. This novel finding highlights an opportunity to improve the quality of declarations, demonstrating that conference officials should ensure that a minimum font size is used by presenters.

Difficulty determining CoI declaration relevance is compounded by the fact that declarations occur at the start of the presentation, and audience members must recall presenter's declarations as potential conflicts arise through the talk. To counter this, presenters could display their declaration at the end of the presentation, as well as at the beginning. To our knowledge, no major conferences currently recommend this practice. Conflicts could also be submitted to a centralised system which auto-populates CoI slides for presenters ensuring appropriate formatting, or, in addition, CoI could also be placed in the conference programme or on-line with the speaker biography. Implementing a centralised system has been proposed by the Institute of Medicine and others. ${ }^{12,13}$ Such a system would address numerous issues, such as the burden on researchers to submit numerous individual declarations, the ambiguity surrounding what should be disclosed, and heterogenous nature of declarations. Fig 2 outlines what a suitable CoI declaration slide may look like, and includes a Quick Response (QR) code which could direct viewers to a centralised database to achieve this aim.

\section{Limitations}

The aim of this study was to highlight the potential importance of additional factors when considering the appropriateness of conference CoI declarations. The main limitations of our study are the single conference assessed, the small number of CoI available for analysis, and the restriction to cardiology presentations. This may limit the external validity of this study's findings and should stimulate further consideration in other medical settings. This research used a pragmatic scoring system which requires further validation. The potential variables considered for this score were limited by the retrospective analysis of presentations. All variables were weighted equally in the scoring system; however, it is likely that some variables are more important than others when declaring conflicts. The weighting of variables and utility of this scoring system could be improved by validating it against a 
Fig 2. Example of a conflict of interest declaration with appropriate formatting and details.

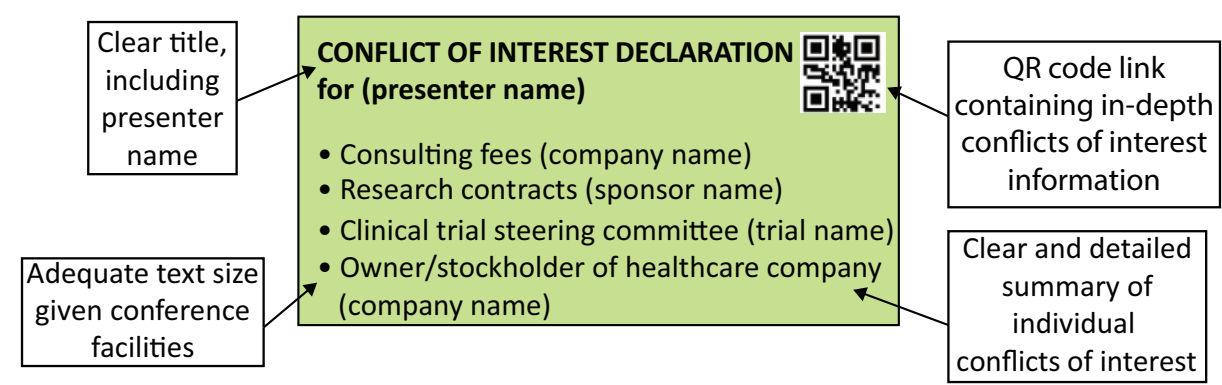

sample audience. This work is not able to comment on the nature of the conflicts declared, and whether they were relevant to their associated presentation. While the relevance of declarations is an important consideration, it was beyond the scope of this study. Finally, it should be noted that the ABPI Disclosure UK database does not give specific information regarding whether payments were received before or after their conference presentation in 2018.

\section{Conclusions}

This study demonstrates potential utility in considering aspects of CoI declarations at conferences other than display time. This work suggests that many CoI declarations at the conference studied are not fit for purpose. While applicability to other conferences/ medical specialties is not certain, all conference organisers should consider what mechanisms are in place to ensure adequate quality of $\mathrm{CoI}$ disclosures. Further research into whether audiences are able to detect and remember potential CoI should be considered, paving the way for policies to mandate declarations of sufficient quality.

\section{References}

1 Dunn AG, Coiera E, Mandl KD, Bourgeois FT. Conflict of interest disclosure in biomedical research: a review of current practices, biases, and the role of public registries in improving transparency. Res Integr Peer Rev 2016;1:1.

2 Brax H, Fadlallah R, Al-Khaled L et al. Association between physicians' interaction with pharmaceutical companies and their clinical practices: A systematic review and meta-analysis. PLoS One 2017;12:e0175493.

3 Lundh A, Lexchin J, Mintzes B, Schroll JB, Bero L. Industry sponsorship and research outcome. Cochrane Database Syst Rev 2017;2: MR000033.
4 Hansen C, Lundh A, Rasmussen K, Hróbjartsson A. Financial conflicts of interest in systematic reviews: Associations with results, conclusions, and methodological quality. Cochrane Database Syst Rev 2019;8:MR000047.

5 Campbell E, Gruen R, Mountford ] et al. A national survey of physician-industry relationships. N Engl J Med 2007;356:1742-50.

6 Silverman E. Everything you need to know about the Sunshine Act. BMJ 2013;347:f4704.

7 Adlington K, Godlee F. Disclosure UK: Transparency should no longer be an optional extra. BMJ 2016;354:i3730.

8 Drazen JM, Van Der Weyden MB, Sahni P et al. Uniform format for disclosure of competing interests in ICMJE journals. N Engl J Med 2009;361:1896-7.

9 Grey A, Avenell A, Dalbeth N, Stewart F, Bolland MJ. Reporting of conflicts of interest in oral presentations at medical conferences: A delegate-based prospective observational study. BMJ Open 2017;7: e017019.

10 Van Lieshout CT, Tijdink JK, Smulders YM. Conflict of interest disclosure slides at the European Society of Cardiology Congress 2016 in Rome: Are they displayed long enough to assess their content? A cross-sectional study. BMJ Open 2018;8:e023534.

11 Taylor S. Eye movements in reading: facts and fallacies. Am Educ Research J 1965;2:187-202.

12 Lichter AS, McKinney R, Anderson T et al. Harmonizing reporting on potential conflicts of interest: a common disclosure process for health care and life sciences: discussion paper. Washington: Institute of Medicine, 2012.

13 Lichter AS, McKinney R. Toward a harmonized and centralized conflict of interest disclosure. JAMA 2012;308:2093-4.

Address for correspondence: Dr C Fielder Camm, Christchurch College, University of Oxford, Oxford OX1 1DP, UK.

Email: christian.camm@chch.ox.ac.uk 